\title{
Complete and enduring response in an elderly patient with repeated recurrent gingival squamous cell carcinoma treated by combined toripalimab and single agent chemotherapy: a case report
}

\author{
Qiuji Wu ${ }^{1,2,3 \#}$, Shuyuan Zhang ${ }^{1,2,3 \#}$, Xinying Hua ${ }^{1,2,3}$, Haijun Yu ${ }^{1,2,3}$, Yahua Zhong ${ }^{1,2,3}$ \\ ${ }^{1}$ Department of Radiation and Medical Oncology, Zhongnan Hospital of Wuhan University, Wuhan, China; ${ }^{2}$ Hubei Key Laboratory of Tumor \\ Biological Behaviors, Zhongnan Hospital of Wuhan University, Wuhan, China; ${ }^{3}$ Hubei Cancer Clinical Study Center, Zhongnan Hospital of Wuhan \\ University, Wuhan, China \\ \#These authors contributed equally to this work. \\ Correspondence to: Yahua Zhong. Department of Radiation Oncology and Medical Oncology, Zhongnan Hospital of Wuhan University, Hubei \\ Key Laboratory of Tumor Biological Behaviors and Hubei Cancer Clinical Study Center, No. 169 Donghu Road, Wuhan 430071, China. \\ Email: doctorzyh73@163.com.
}

\begin{abstract}
Combined immune checkpoint inhibitor with platinum-based doublet chemotherapy brought about significant improvement in overall survival as the first line treatment in metastatic and recurrent head and neck cancer patients. However, in elderly patients with relatively poor performance status, these regimens might not be well tolerated. Therefore, we evaluated the safety and effect of combined immunotherapy and single agent chemotherapy in an elderly patient with recurrent head and neck squamous cell carcinoma. A 78-year-old male patients with a repeatedly recurrent gingival squamous cell carcinoma was admitted in our institute. Previously this patient underwent two consecutive surgical resections of recurrent tumors before the second rapid recurrence that resulted in extended tumor mass and lymph node metastasis. As the patient was in a relatively poor performance status (performance status $=2$ ), immunotherapy with PD-1 antibody (Toripalimab) combined with single agent chemotherapy (two cycle with albumin-bound paclitaxel and then six cycles with gemcitabine) was administrated, which led to clinical complete response after 8 cycles of treatment. The patient continued 4 cycles of maintenance immunotherapy with Toripalimab until the outbreak of the coronavirus disease 2019. The treatment was well tolerated and the patient remained free from disease until the last follow up by June 2020, 16 months after the initiation of treatment. The success in this case indicated that the combination of anti-PD-1 antibody and single chemotherapy agent may also be effective as well as safe in elderly patients with recurrent head and neck squamous cell carcinoma.
\end{abstract}

Keywords: Recurrent HNSCC; PD-1 inhibitor; chemotherapy; elderly patient; case report

Submitted Jul 27, 2020. Accepted for publication Aug 28, 2020.

doi: 10.21037/apm-20-1485

View this article at: http://dx.doi.org/10.21037/apm-20-1485

\section{Introduction}

For patients with locally incurable recurrent head and neck squamous cell carcinoma (RHNSCC), the EXTREME regimen (cetuximab in combination with platin and 5 -fluorouracil) was the standard of care. However, response rate to this systemic therapy was limited, with the median progression-free survival being only 5.6 months and the median overall survival being only 10.1 months (1). The KEYNOTE-048 phase III clinical trial has demonstrated a significant improvement of overall survival in patients 
treated with pembrolizumab and doublet chemotherapy as compared with those received EXTREME regimen (2). Importantly, combined immunotherapy with chemotherapy yielded enduring response but did not increase the frequency and severity of treatment-associated side effects (3). Although pembrolizumab combined with platinum and fluorouracil in RHNSCC has shown significant advantage over classic chemotherapy, the study has excluded older patients or patients with poorer performance status. Here, we report a case of 78-yearold male patient with repeated recurrent HNSCC who experienced a dramatic response and excellent local control after treatment with anti-PD-1 and single agent chemotherapy.

We present the following article in accordance with the CARE reporting checklist (available at http://dx.doi. org/10.21037/apm-20-1485).

\section{Case presentation}

A 78-year-old man with recurrent gingival squamous cell carcinoma presented to our institution on March $12^{\text {th }} 2019$, after the second relapse of his original disease. On admission, the patient complained of swelling and pain on the right cheek. For medical history, we noted an arterial hypertension that was well controlled with antihypertensive drugs. He had no other medical history and denied any smoking or drinking history. No family history of cancer was reported.

Previously, the patient was hospitalized in Wuhan Union Hospital of China in August 2018 for the reason of the right mandibular mass with pain for 3 months. On September 18,2018, MR examination of the head and neck showed a $4.5 \mathrm{~cm} \times 3.6 \mathrm{~cm} \times 3.7 \mathrm{~cm}$ gingival mass invading into the right inferior alveolar bone and the body of mandible. Initially, he underwent a curettage of right mandibular mass instead of radical operation due to a deep venous thrombus of the right lower extremity. The pathohistological examination revealed a well-to-moderately differentiated squamous cell carcinoma. Following the placing of inferior vena cava filter, the patient received the right oral and maxillofacial tumor resection with right cervical lymph node dissection as well as a flap reconstruction. Postoperative pathological diagnosis was a well-tomoderately differentiated squamous cell carcinoma with invasion of mandible and involvement of peripheral striated muscles. No positive lymph node (0/19) was found. $\mathrm{He}$ did not search for further treatment except for traditional
Chinese medicine.

However, in November of 2018, he experienced the first local recurrence in the right maxillary gingival area in the form of a painful mucous leukoplakia measuring about $3.5 \mathrm{~cm} \times 3.0 \mathrm{~cm}$. He received a second surgical resection of the maxillary tumor with a flap reconstruction in Wuhan Union Hospital of China. Postoperative pathological examination demonstrated a keratinized well-to-moderately differentiated squamous cell carcinoma of the right superior gingiva that invaded the right maxilla. Unfortunately, not long after the surgery, the patient suffered from swelling and pain in his right buccal area in January 2019. A neoplasm with cutaneous fistula developed in the right buccal and submandibular area. The biopsy of the neoplasm made in Wuhan Tongji Hospital turned out to be a squamous cell carcinoma.

Thereafter, the patient turned to our institute for further treatment on March $12^{\text {th }} 2019$. On admission, the patient was thin and weak with a performance score about 2 . The physical examination found a palpable hard mass about $3.5 \mathrm{~cm} \times 3.5 \mathrm{~cm}$ in the right buccal area. In addition, three crateriform neoplasm with fistula and skin erosion accompanied by marked purulent secretion and errhysis were found near the right submandibular area and mandibular angles. No obvious palpable enlarged lymph node was found in bilateral necks and the supraclavicular regions. A positron emission tomographyCT (PET-CT) revealed a right submandibular mass of $4.5 \mathrm{~cm} \times 3.5 \mathrm{~cm}$ with SUV of 7.6-18.3 indicating tumor recurrence (Figure 1A). There were multiple right cervical lymph node metastases. Otherwise, PET-CT did not show clear sign of distant metastasis. Considering large extension of his disease complicated with fistula and infections as well as his poor performance status, we recommended to him systemic treatment with less toxic single agent chemotherapy plus immunotherapy. Then he received the first cycle of albumin-bound paclitaxel every 21 days (400 mg d1, q21d) and one anti-PD-1 antibody, Toripalimab every 21 days (240 mg d1, q21d) on March 14, 2019, and the second cycle was performed on April 4, 2019. Meanwhile, antibiotics and daily debridement were also applied. Importantly, the patient presented a significant regression of his tumor and recovery from infections. The pain was relieved and the performance status was also improved. The treatment was well tolerated except for a grade II leucopenia and a grade I gastrointestinal reaction. However, the patient refused to continue albumin-bound paclitaxel due to economic issues. Therefore, we changed the regimen to gemcitabine 
A
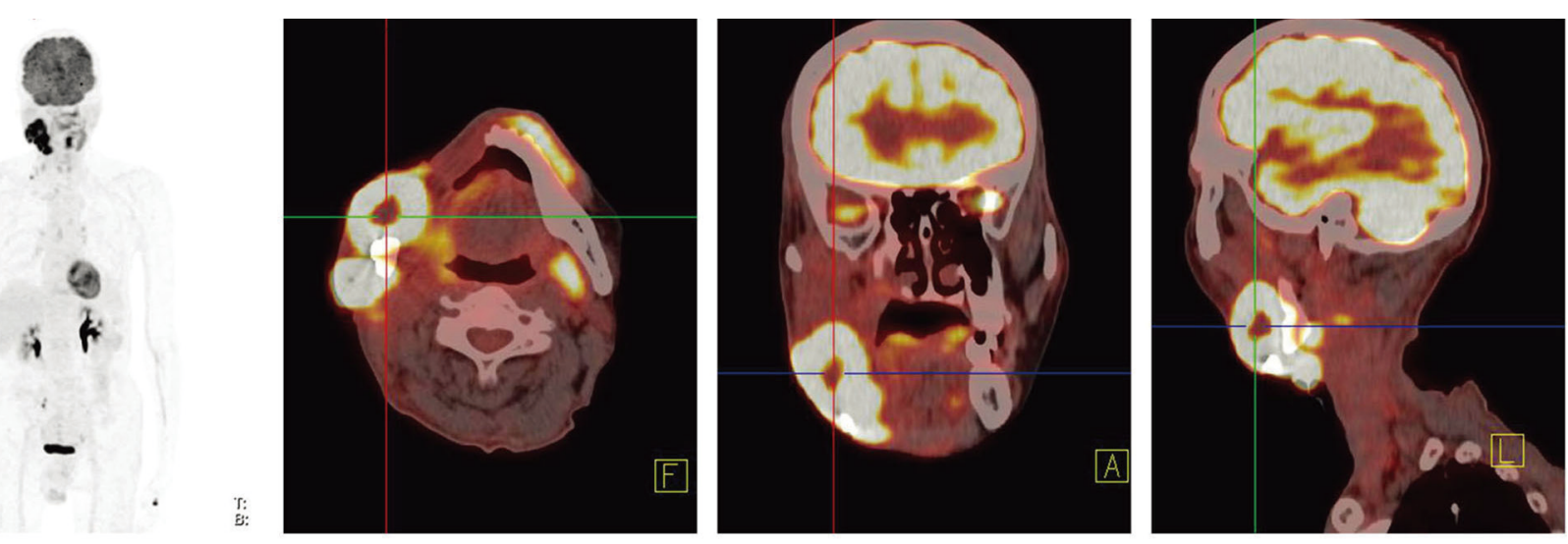

B
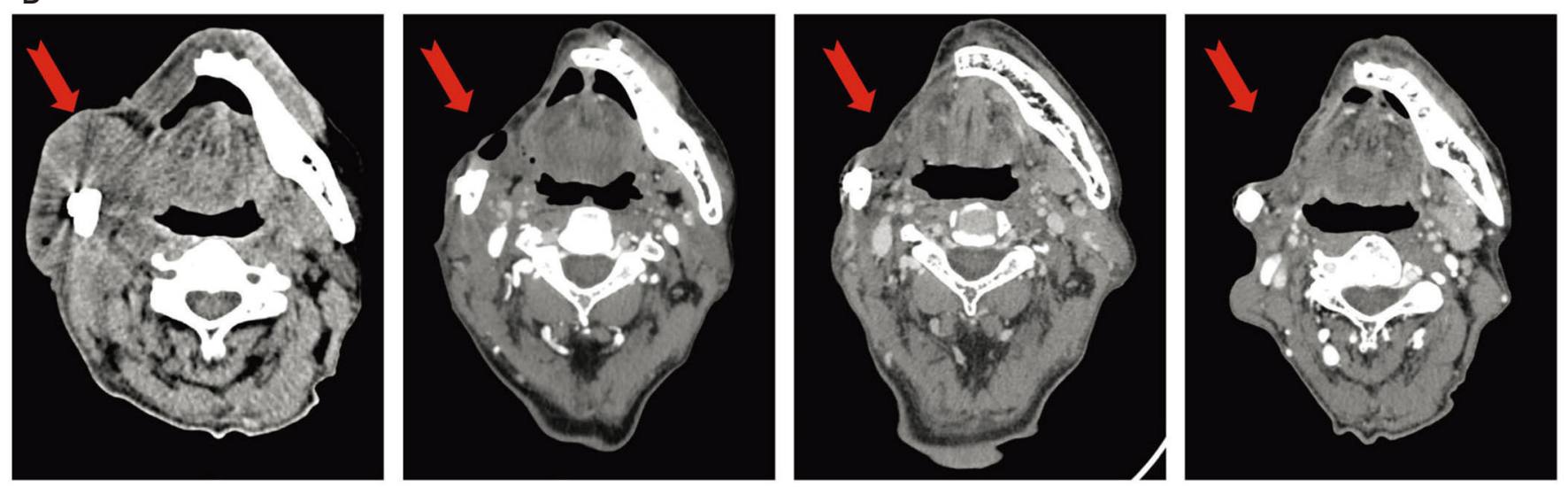

C

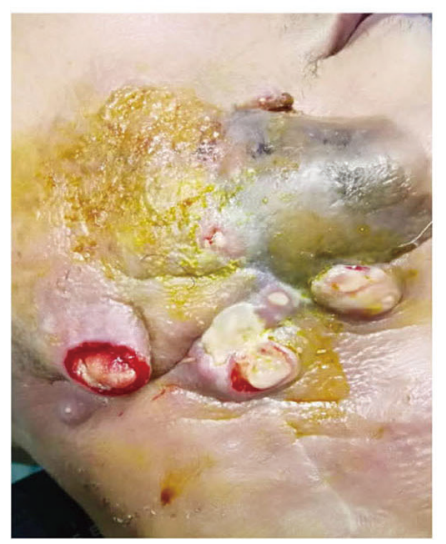

Pre-treatment

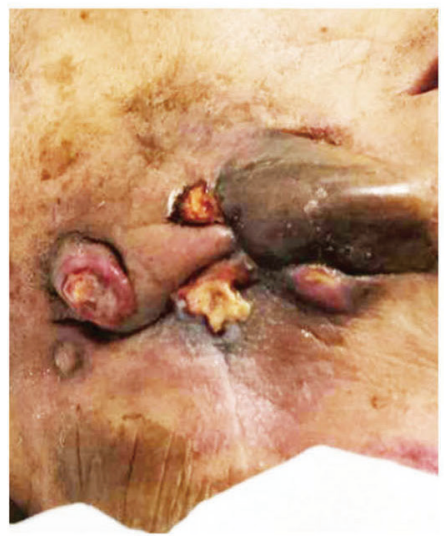

$\mathrm{ICI}+$ chemotherapy 2 cycles

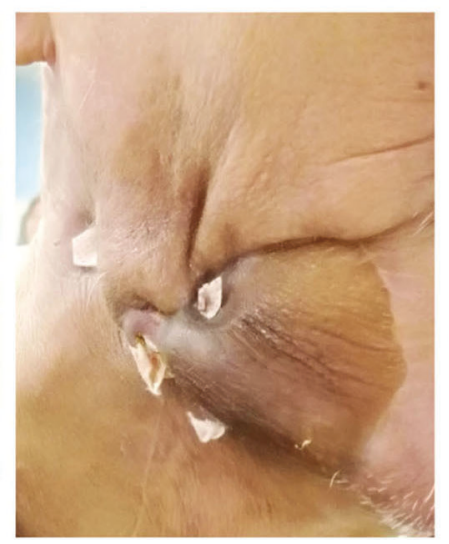

$\mathrm{ICl}+$ chemotherapy 8 cycles

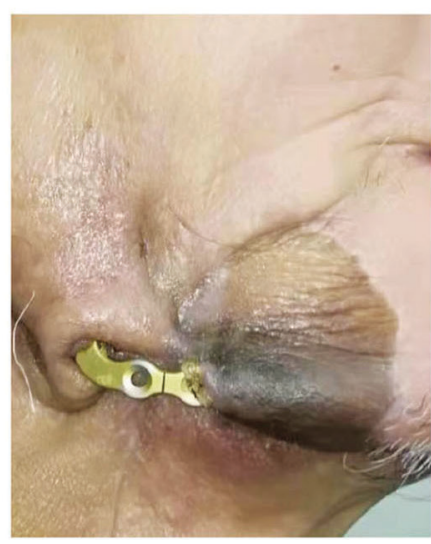

$\mathrm{ICl}$ maintenance 4 cycles

Figure 1 Pre-treatment PET/CT examination and disease responses during the systemic treatment. (A) PET images and PET/CT images of the relapsed tumor and lymph nodes. (B) CT images and (C) clinical morphological manifestation recorded at pre-treatment, after 2 cycles and 8 cycles of combined treatment and after 4 cycles of maintenance immunotherapy, respectively, were shown. Red arrows indicate the area of the tumor mass. ICI, immunocheckpoint inhibitor. 


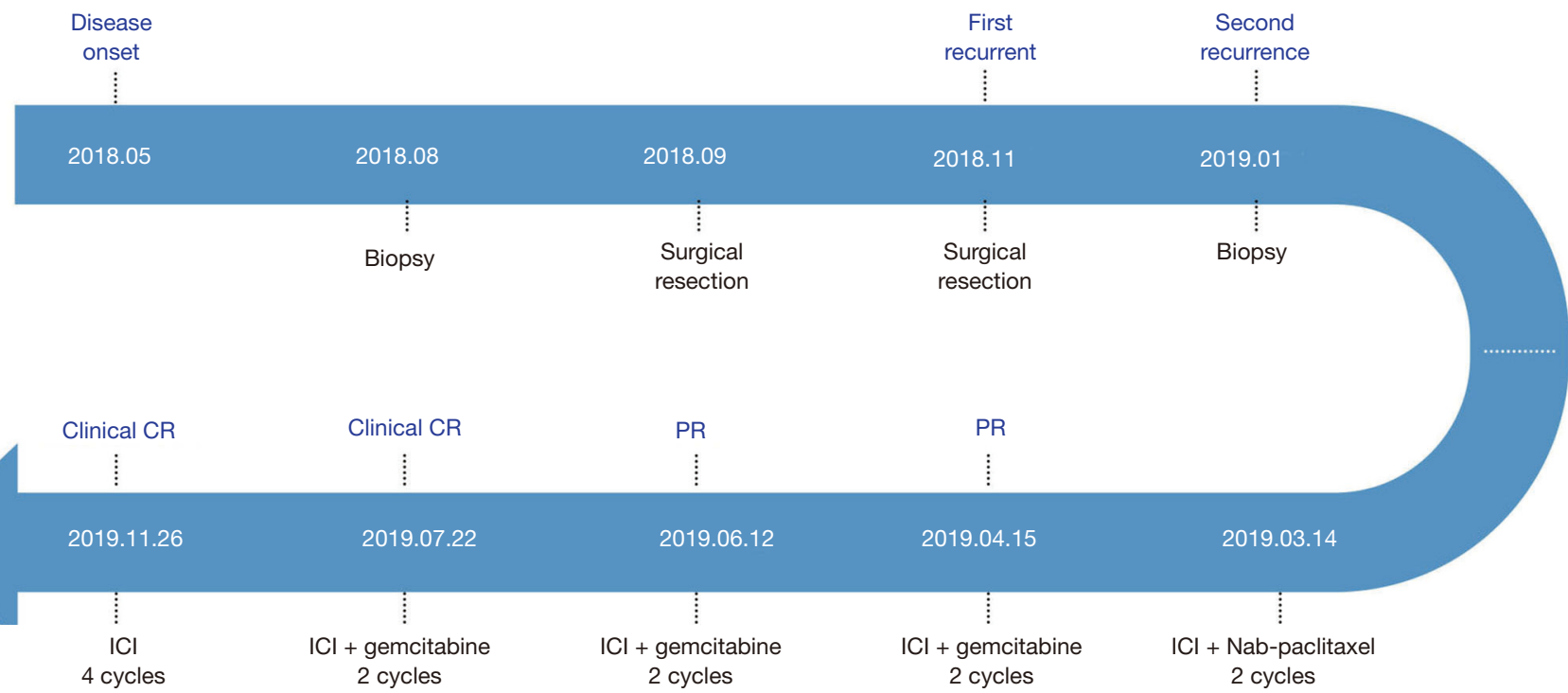

Figure 2 Time line summary of treatments. CR, complete response; PR, partial response; ICI, immunocheckpoint inhibitor.

(1.5 g d1, d8; q21d) and Toripalimab (240 mg d1, q21d). The patients further received 6 cycles of treatment from April 24, 2019 to August 15, 2019 during which time the tumor continued to regress and the fistula disappeared. Contrast-enhanced CT scans showed no significant residual of tumor or lymph node. The response evaluation was a clinical complete response.

From September 5, 2019 to November 26, 2019, he received 4 cycles of maintenance treatment with Toripalimab every 21 days ( $240 \mathrm{mg} \mathrm{d} 1$, q21d) that was well tolerated. The treatment was discontinued due to the coronavirus disease 2019 pandemic (Figure 1B,C). However, the patient remained free of disease until the last follow up by June 2020, 16 months after the initiation of treatment without obvious toxicities (Figure 2).

All procedures performed in studies involving human participants were in accordance with the ethical standards of the institutional and/or national research committee(s) and with the Helsinki Declaration (as revised in 2013). Written informed consent was obtained from the patient.

\section{Discussion}

The revolutionary phase III study KEYNOTE-048 compared pembrolizumab alone, pembrolizumab plus a platinum and 5-fluorouracil, or cetuximab plus a platinum and 5 -fluorouracil in patients with untreated locally incurable recurrent or metastatic HNSCC (RMHNSCC). The study found that pembrolizumab with chemotherapy significantly improved overall survival compared to cetuximab with chemotherapy in the total population (13.0 vs. 10.7 months, HR 0.77; 95\% CI: 0.63-0.93, $\mathrm{P}=0.0034$ ) and showed more pronounced advantage in patients with a PD-L1 combined positive score (CPS) no less than 20 (14.7 vs. $11.0,0.60$; $95 \%$ CI: $0.45-0.82, \mathrm{P}=0.0004)$ and in patients with a PD-L1 CPS no less than 1 (13.6 vs. 10.4, 0.65; $95 \%$ CI: $0.53-0.80, \mathrm{P}<0.0001)$ (2). This was the first trial that demonstrated a significant improvement of overall survival in the first line treatment in locally incurable recurrent or metastatic HNSCC that prompted the quick approval of pembrolizumab in this setting. However, this study only included patient with good performance status $(\mathrm{PS} \leq 1)$ and all patient were no more than 68 years old (2). The value of immunotherapy combined with chemotherapy in older patients ( $\geq 75$ years old) or patients with poor performance status ( $\mathrm{PS} \geq 2$ ) was not clear.

In this case, combined Toripalimab and single-agent chemotherapy (albumin-bound paclitaxel or gemcitabine) showed clinical complete response and moderate toxicities in a 78-year old patient with a PS score of 2 that suffered from a repeated recurrence of a gingival squamous cell carcinoma. Importantly, he had previously received two surgical resections of the recurrent disease but experienced rather short disease-free periods, indicating a welldifferentiated but highly aggressive type of cancer. By the time of the latest recurrence, the patient already developed multiple tumor necrosis, fistula, cervical lymph node metastasis and local infections. For immunotherapy, since 
pembrolizumab was economically unavailable for this patient who have underwent extensive previous treatments, we recommended him Toripalimab, a recombinant, humanized programmed death receptor-1 (PD-1) monoclonal antibody that had been approved in unresectable or metastatic melanoma which had failed in previous systemic therapy in 2018 in China (4). More clinical trials testing Toripalimab in head and neck cancers are ongoing (NCT03952065, NCT03854838, NCT04164238). For chemotherapy we recommended active but less toxic agents that did not require corticosteroid pretreatment. After the treatment, the patient achieved clinical complete response and his quality of life was improved significantly. Meanwhile, he did not experience any 3-4 grade treatment-associated adverse events. This case report suggested that Toripalimab in combination with single-agent chemotherapy might be effective and safe for patients with RHNSCC, although it was still important to determine which chemotherapy agent would show better synergistic effect with immunotherapy.

The case also indicated that simultaneous antibiotic use was necessary in the control of tumor-associated infections and did not affect the treatment efficacy. Indeed, there were a few studies showing previous use of antibiotics might dampen the effect of immunotherapy (5-8). For future treatments, we consider that local radiation therapy and maintenance immunotherapy might be one choice, according to the physical and disease status of the patient.

Given the success that was observed, the case also had several shortcomings. First, PD-L1 expression status was not known that should be a putative predictor of immunotherapy $(9,10)$. Second, the follow up time was still short and further follow-up are needed. Additionally, challenges still exist regarding salvage treatment in case of relapse.

In conclusion, in this case report the combination of Toripalimab and single-agent chemotherapy showed excellent response and good tolerance in the first-line treatment of an older patient with RHNSCC. Perspective randomized trial studying the efficacy of Toripalimab in combination with single-agent chemotherapeutic agent as the first-line treatment in patients with RHNSCC would be valuable.

\section{Acknowledgments}

Funding: This work was supported by the National Natural Science Foundation Youth Project (grant number: 81803061).

\section{Footnote}

Reporting Checklist: The authors have completed the CARE reporting checklist. Available at http://dx.doi.org/10.21037/ apm-20-1485

Conflicts of Interest: All authors have completed the ICMJE uniform disclosure form (available at http://dx.doi. org/10.21037/apm-20-1485). The authors have no conflicts of interest to declare.

Ethical Statement: The authors are accountable for all aspects of the work in ensuring that questions related to the accuracy or integrity of any part of the work are appropriately investigated and resolved. All procedures performed in studies involving human participants were in accordance with the ethical standards of the institutional and/or national research committee(s) and with the Helsinki Declaration (as revised in 2013). Written informed consent was obtained from the patient for publication of this study and any accompanying images.

Open Access Statement: This is an Open Access article distributed in accordance with the Creative Commons Attribution-NonCommercial-NoDerivs 4.0 International License (CC BY-NC-ND 4.0), which permits the noncommercial replication and distribution of the article with the strict proviso that no changes or edits are made and the original work is properly cited (including links to both the formal publication through the relevant DOI and the license). See: https://creativecommons.org/licenses/by-nc-nd/4.0/.

\section{References}

1. Vermorken JB, Mesia R, Rivera F, et al. Platinum-based chemotherapy plus cetuximab in head and neck cancer. $\mathrm{N}$ Engl J Med 2008;359:1116-27.

2. Burtness B, Harrington KJ, Greil R, et al. Pembrolizumab alone or with chemotherapy versus cetuximab with chemotherapy for recurrent or metastatic squamous cell carcinoma of the head and neck (KEYNOTE-048): a randomised, open-label, phase 3 study. Lancet 2019;394:1915-28.

3. Cohen EEW, Bell RB, Bifulco CB, et al. The Society for Immunotherapy of Cancer consensus statement on immunotherapy for the treatment of squamous cell carcinoma of the head and neck (HNSCC). J Immunother Cancer 2019;7:184. 
4. Keam SJ. Toripalimab: First Global Approval. Drugs 2019;79:573-8.

5. Pinato DJ, Howlett S, Ottaviani D, et al. Association of Prior Antibiotic Treatment With Survival and Response to Immune Checkpoint Inhibitor Therapy in Patients With Cancer. JAMA Oncol 2019;5:1774-8.

6. Wilson BE, Routy B, Nagrial A, et al. The effect of antibiotics on clinical outcomes in immune-checkpoint blockade: a systematic review and meta-analysis of observational studies. Cancer Immunol Immunother 2020;69:343-54.

7. Schett A, Rothschild SI, Curioni-Fontecedro A, et al. Predictive impact of antibiotics in patients with advanced non small-cell lung cancer receiving immune checkpoint

Cite this article as: $\mathrm{Wu} \mathrm{Q}$, Zhang $\mathrm{S}$, Hua $\mathrm{X}$, Yu H, Zhong $\mathrm{Y}$. Complete and enduring response in an elderly patient with repeated recurrent gingival squamous cell carcinoma treated by combined toripalimab and single agent chemotherapy: a case report. Ann Palliat Med 2021;10(6):6968-6973. doi: 10.21037/ apm-20-1485 inhibitors: Antibiotics immune checkpoint inhibitors in advanced NSCLC. Cancer Chemother Pharmacol 2020;85:121-31.

8. Huang XZ, Gao P, Song YX, et al. Antibiotic use and the efficacy of immune checkpoint inhibitors in cancer patients: a pooled analysis of 2740 cancer patients. Oncoimmunology 2019;8:e1665973.

9. Patel SP, Kurzrock R. PD-L1 Expression as a Predictive Biomarker in Cancer Immunotherapy. Mol Cancer Ther 2015;14:847-56.

10. Zou W, Wolchok JD, Chen L. PD-L1 (B7-H1) and PD-1 pathway blockade for cancer therapy: Mechanisms, response biomarkers, and combinations. Sci Transl Med 2016;8:328rv4. 